\title{
A Multi-touch Tool for Co-creation
}

\author{
Geke D.S. Ludden and Tom Broens
}

\author{
Novay, Brouwerijstraat 1, P.O. Box 589, 7500 AN, Enschede, The Netherlands \\ \{Geke. Ludden, Tom.Broens\} atelin.nl
}

\begin{abstract}
Multi-touch technology provides an attractive way for knowledge workers to collaborate. Co-creation is an important collaboration process in which collecting resources, creating results and distributing these results is essential. We propose a wall-based multi-touch system (called CoCreate) in which these steps are made easy due to the notion of connected private spaces and a shared co-create space. We present our ongoing work, expert evaluation of interaction scenarios and future plans.
\end{abstract}

Keywords: multi-touch, wall, interaction scenario, CoCreate.

\section{Introduction}

Multi-touch technology gains a lot of attention in research and industry. Bill Buxton gives a nice summary of work in this area [1]. Multi-touch technology is used for phones (e.g. Iphone), computer input interfaces (e.g. track pads) and surfaces (e.g. tables and walls). Multi-touch technology allows multiple users to interact with a surface simultaneously and therefore seems suitable to support the co-creation processes of knowledge workers. Despite this potential, the number of tools using multi-touch technology for co-creation is still limited. In this paper we investigate the feasibility of a wall-based multi-touch tool for co-creation.

Co-creation is all about creating and sharing. In general we distinguish three phases in a co-creation process: (i) collecting public and private resources of participants (ii) create a shared result and (iii) distribute this result to the participants. For an optimal process all phases should be supported.

Much of the existing co-creation tools have a table form factor [e.g., 2, 3, 4]. Rogers and Lindley [5] investigated differences in collaborating using vertical and horizontal displays. They concluded that a table form factor facilitated collaboration better. However, the setup they used may have negatively influenced their findings for collaborating using a vertical display. We doubt whether a horizontal display is the most suitable for co-creation. Analogous to stand-up (or 'scrum') meetings that are claimed to be more efficient [6], a wall-based system stimulating standing sessions might result in a quicker and more creative process compared to sitting sessions at a table. Furthermore, intrinsically a wall has the same orientation for all participants, which eases the creation process. Additionally, in a remote table-based setting, such as Digitable [2] creating a feeling of connectedness by showing a camera feed of the remote participants, which is the de-facto standard, is more awkward due to the face-camera orientation. 
Hence, we propose a wall-based CoCreate system in which collecting resources and sharing the result is made easy due to the notion of connected private spaces and a shared co-create space. In the remainder of this paper we discuss the design of the CoCreate tool, and an initial feasibility evaluation, conclusions and our future plans.

\section{Design}

We created interaction scenarios for co-creation using a multi-touch wall at one location ('co-located') and at a distance ('at a distance'). For these scenarios, we envision a multi-touch wall with a display area of $185 \times 104 \mathrm{~cm}$.

\section{1 'Co-located' Co-creation Using Multi-touch}

Generally, the 'co-located' CoCreate tool comprises both a co-creation space and personal working spaces for current users. The personal working space contains links to, for example, Flickr or Delicious accounts, the user's desktop, and his or her frequently used folders. The following figure illustrates how this tool will typically be used.

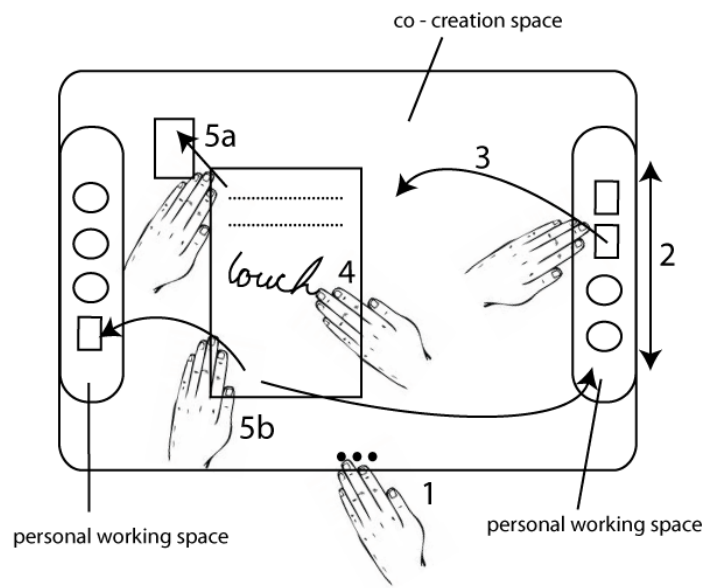

(1) Open CoCreate from the application dock. Personal working spaces are opened for all users. (2) Browse through personal working space. (3) Drag article into the co-creation space. The article enlarges. (4) Write a comment on the article. (5a) Put aside the article, article minimizes. (5b) Drag the article into personal spaces.

\subsection{Co-creation Using Multi-touch 'at a distance'}

The CoCreate tool 'at a distance' is aimed at supporting remote knowledge workers to create together. Please note that multi-touch technology is not essential in this scenario. However, this scenario provides an additional way to use a multi-touch wall. The tool typically shows a video of a remote person, calendars of all current users, a personal working space and a co-creation space. The following figure provides an example of how such a tool could be used. 


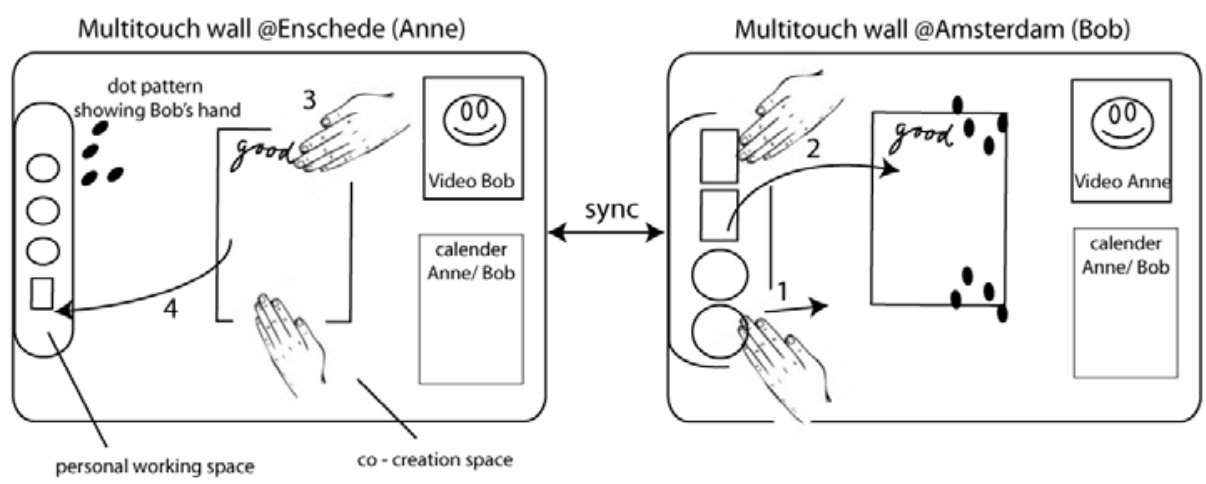

(1) Enlarging personal working space. (2) Drag document to co-creation space, an enlarged document appears on both walls. While Bob is manipulating items on his wall, Anne sees a dot pattern representing the location of Bob's hand on her wall. The two can talk about the document using the video connection. (3) Anne makes a note on the document, now Bob sees a dot pattern representing Anne's hand. (4) Anne drags the document into her personal space.

\section{Evaluation}

The feasibility of our CoCreate tools was evaluated by a group of experts. To do so, the scenarios as described above were posted on a website together with two comics illustrating situations in which multi-touch displays could help people to work together more efficiently. Visitors of our website were asked to indicate to what end they agreed to three statements about the specified interaction choosing from five options: 'strongly agree', 'agree', 'neither agree nor disagree', 'disagree' and 'strongly disagree'. The statements were: (Q1)'I like the features of this concept'; (Q2)'I think this concept can help people to work together', (Q3)'I think the interaction expressed in this scenario will work well'. Furthermore, we asked for additional remarks about the scenarios.

Seven experts that were selected based on their expertise on designing for interaction. (6 male, 1 female, aged 22-31) evaluated the interaction scenarios. Generally, most respondents evaluated both scenarios positively. For both scenarios, as a response to Q1 and Q2, all respondents selected either 'agree' or 'agree completely'. Responses on Q3 were somewhat less positive, 43\% of the responses for the 'colocated' scenario and $29 \%$ of the responses for the 'at a distance' scenario were 'neither agree nor disagree'. Other responses were either 'agree' or 'completely agree'. Although the responses on scales generally were positive, the remarks that respondents made in addition to their responses on scales indicate that some of them had doubts about the added value of using a multi-touch wall over traditional working methods. Also, it was mentioned that using CoCreate would require new ways of organizing meetings. One respondent mentioned privacy issues coming from the fact that all users can see each others' personal spaces. (This of course only applies to the 'colocated' scenario.) Some respondents mentioned that they would see more use to simpler applications on a multi-touch wall. For the 'at a distance' scenario, respondents did not agree about the value of seeing the other persons' actions as dots representing a hand. One respondent suggested using the image of a shadow of a hand instead of 
dots. These are all typically issues we would like to test in a pilot set-up. At the same time, we would like to understand the impact of this concept on day-to-day work. Therefore, a working prototype of the proposed concept will be implemented in a real work environment in industry.

\section{Conclusions and Future Work}

It must be noted that for our 'at a distance' scenario, a multi-touch wall is not the only way to solve the issue of sharing interfaces. Other projection tools such as Lucid Lab's Virtual Desktop [7] also allow co-creation. However, for the 'co-located' scenario, multi-touch is essential to allow multiple persons to work at the same interface. To implement and test the ideas laid out above, we are currently developing both hardware and software. A multi-touch wall implementing CoCreate functionality will be created in cooperation with Soco Amsterdam [8]. The wall will rely on Frustrated Total Internal Reflection (FTIR) technology and will feature a display area of $185 \mathrm{x}$ $104 \mathrm{~cm}$. At the same time, we are working on improvements of the scenarios, more specifically we are working out the interaction with personal spaces especially in situation where more than two people use the screen.

\section{References}

1. Bill Buxton, http: //www.billbuxton.com/multitouchoverview.html

2. Pauchet, F., Coldefy, L., Lefebvre, S., Louis Dit Picard, A., Bouguet, L., et al.: Mutual awareness in collocated and distant collaborative tasks using shared interfaces. In: Baranauskas, C., Palanque, P., Abascal, J., Barbosa, S.D.J. (eds.) INTERACT 2007. Part. I. LNCS, vol. 4662, pp. 59-73. Springer, Heidelberg (2007)

3. Shen, C., Vernier, F.D., Forlines, C., Ringel, M.: DiamondSpin: an extensible toolkit for around-the-table interaction. In: ACM Conference on Human Factors in Computing Systems (CHI), pp. 167-174. ACM Press, New York (2004)

4. Hunter, S., Maes, P.: Wordplay: a table-top interface for collaborative brainstorming and decision making. Submitted to IEEE TableTop Collaborative Surfaces (2008)

5. Rogers, Y., Lindley, S.: Collaborating around vertical and horizontal large interactive displays. Interacting with Computers 16, 1133-1152 (2004)

6. Vizdos, M.: Opening communication within a scrum team. Methods \& Tools 16, 31-35 (2008), http: / / www . methodsandtools . com/PDF/mt200803.pdf

7. Elsen, C., Leclercq, P.: "SketSha" - The sketch power to support collaborative design. In: Luo, Y. (ed.) CDVE 2008. LNCS, vol. 5220, pp. 20-27. Springer, Heidelberg (2008)

8. SocoAmsterdam, http://www. socoamsterdam.nl 